\title{
Are Polar Rings Indeed Polar?
}

\author{
K. I. Smirnova ${ }^{1^{*}}$ and A. V. Moiseev ${ }^{2}$ \\ ${ }^{1}$ Ural Federal University, Ekaterinburg, 620002 Russia \\ ${ }^{2}$ Special Astrophysical Observatory, Russian Academy of Sciences, Nizhnii Arkhyz, 369167 Russia \\ Received August 24, 2013; in final form, September 20, 2013
}

\begin{abstract}
We have considered polar ring galaxy candidates, the images of which can be found in the SDSS survey. The sample of 78 galaxies includes the most reliable candidates from the SPRC and PRC catalogs, some of which already have kinematic confirmations. We analyze the distributions of studied objects by the angle between the polar ring and the central disk, and by the optical diameter of the outer ring structures. In the vast majority of cases, the outer structures lie in the plane close to polar (within $10^{\circ}-20^{\circ}$ ) which indicates the stability of the corresponding orbits in the gravitational potential of the halo. Moderately inclined outer structures are observed only in about $6 \%$ of objects which probably indicates their short lifetime. In such an unstable configuration, the polar ring would often cross the disk of the galaxy, being smaller than it in the diameter. We show that the inner polar structures and outer large-scale polar rings form a single family in the distribution of diameters normalized to the optical size of the galaxy. At the same time, this distribution is bimodal, as the number of objects with $d_{\text {ring }}=(0.4-0.7) d_{\text {disk }}$ is small. Such a shape of size distribution is most likely due to the fact that the stability of polar orbits in the inner regions of galaxies is maintained by the bulge or the bar, while in the outer regions it is provided by the spheroidal (or triaxial) halo.
\end{abstract}

DOI: $10.1134 / \mathrm{S} 1990341313040019$

Keywords: galaxies: peculiar-galaxies: photometry

\section{INTRODUCTION}

Polar ring galaxies (PRG) simultaneously demonstrate rotation about two axes: apart from the central stellar disk, an extended structure composed of stars and gas, called a polar ring or a polar disk, rotates in the plane perpendicular or highly inclined to it. Prior to the release of our paper in 2011 [1], virtually all the studies of such galaxies referred to the objects presented in the catalog of Whitmore et al. [2]. This catalog (Polar Rings Catalog, PRC), based on the study of photographic data, is divided into four parts and includes 157 objects. However, only six of them ("type A") were kinematically confirmed at the moment of publication. The authors have attributed 27 galaxies to the type B (good candidates). A major part of the catalog was comprised of peculiar objects with various signs of interaction with the environment, only possibly related to the phenomenon of polar rings.

To relegate a galaxy to the class of PRGs, the following has to be fulfilled according to Whitmore et al. [2]:

\footnotetext{
*E-mail: arashu@rambler.ru
}

- the presence in the galaxy of two sub-systems rotating in almost orthogonal planes and having comparable rotation velocities;

- both kinematically isolated subsystems should have matching systemic velocities, i.e., to be an object with a common center rather than a random projection of the two galaxies on the line of sight, their photometric centers evidently have to match;

- the polar structure (a ring or a disk) has to be comparable in size with the central galaxy and look relatively flat.

During the subsequent years of study of the PRC objects, only about two dozens of cases obtained kinematic confirmations that these galaxies indeed belong to the PRGs. This means that the presence of matter in polar orbits was verified using optical spectroscopy and radio interferometry in the line of $\mathrm{H} \mathrm{I}$.

In 2011 we have published [1] a catalog of the polar ring candidates, consisting of 275 candidates selected from the images of the Sloan Digital Sky Survey (SDSS DR7) mainly owing to the use of GalaxyZoo 

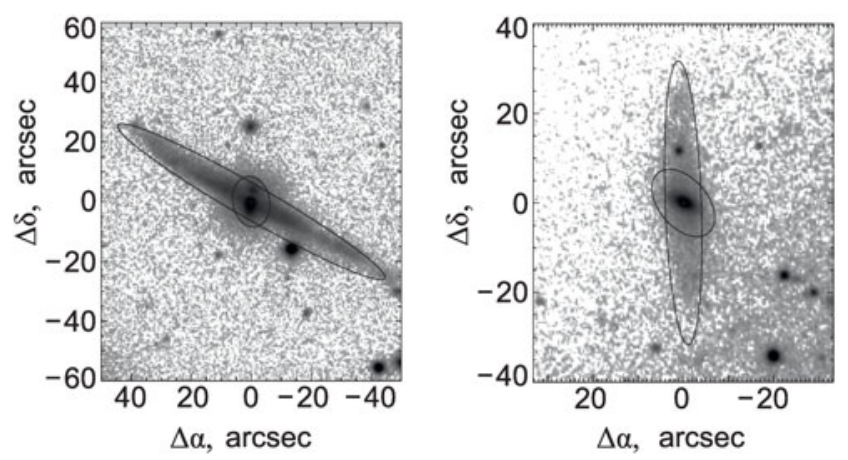

Fig. 1. Image of the SPRC 42 (left) and SPRC 50 (right) galaxies, the sum of five SDSS filters in the logarithmic intensity scale. The ellipses show the accepted orientations of the outer regions of the disk and the polar structure.

project data. In this catalog (SDSS-based Polar Ring Catalogue, SPRC), we also applied a conventional separation of objects into four types based on their optical morphology only:

- the best candidates (70 galaxies);

- good candidates, including possible random projections (115 galaxies);

- related objects with strongly warped disks (53 galaxies);

- rings viewed nearly face-on (37 galaxies).

Several scenarios of the formation of PRGs were developed. All of them are in one or another way related with the interaction of the central galaxy with its environment (see $[3,4]$ and references therein). One scenario is a merger of two galaxies with mutually orthogonal disks that occurs at relatively low mutual velocities. Calculations show that a large part (about $3 / 4$ ) of polar rings is most likely produced from the accretion by a large galaxy of the matter of its gasrich companion having the corresponding direction of its orbital rotation momentum. This requires very specific initial conditions $[3,5]$. The capture of gas itself may naturally occur at any orbital orientation of the "donor" companion. However, if the gas ring formed this way is inclined at a small angle to the stellar disk of the galaxy, then in a few revolutions under the influence of the gravity of the disk, the ring will fall in the plane of the galaxy due to precession. At the same time, the polar (i.e. almost perpendicular) orientation of the disk and the ring is stable, hence the ring will survive a lot of revolutions. An important

\footnotetext{
${ }^{1}$ www.galaxyzoo.org
}

stabilizing role is played here by the dark halo in the event when it has a flattened or elongated shape.

The scenario of formation of some polar rings in the process of gas accretion from the filaments of the intergalactic medium (the so-called "cold accretion") is recently gaining popularity [6-8]). This may explain the existence of the largest polar structures in which the mass of the polar component is equal to or greater than the mass of the central galaxy. It is important to note that in itself, cold accretion is a natural stage undergone by galaxies when they are gaining their baryon mass. But only in some specific cases it is possible to notice the traces of this process.

To date, PRGs are among the few types of objects allowing to directly estimate the shape of the dark halo owing to the ability to measure the rotation curve in two perpendicular planes.

In order to understand exactly what mechanism led to the formation of a PRG in each particular case, as well as to address the issue of stability of polar structures, it is useful to consider the statistical distributions of their main parameters, such as the relative size and inclination to the plane of the central disk. Are only the orbits orthogonal to the disk longlived? How often do polar rings cross the stellar disk of the host galaxy? The statistics of the parameters of polar components in galaxies was earlier considered in [9] on a small sample of 27 PRC galaxies. Now we can significantly increase the number of studied objects, using a homogeneous enough set of observations-the SDSS images. Our paper is dedicated to this.

\section{CANDIDATE SAMPLE}

To study the statistics of the PRG parameters, it is important to have the maximally uniform initial material. Our sample included 70 SPRC galaxies classified in [1] as "the best candidates," i.e., bearing the largest similarity with classic PRGs. To these, we have added two galaxies classified as "rings oriented face-on": SPRC 241, SPRC 260, which have already been kinematically confirmed to belong to the PRGs [10]. We have also included in the sample the PRC catalog objects for which, on the one hand, there are kinematic confirmations of belonging to the PRG class and, on the other hand, there are SDSS/DR8 images. Such galaxies were only six. In total, the sample consists of 78 galaxies with redshifts in the range of $z=0.003-0.17$. It is important that the analyzed images have similar limits of surface brightness, since we use a common image source, SDSS. 

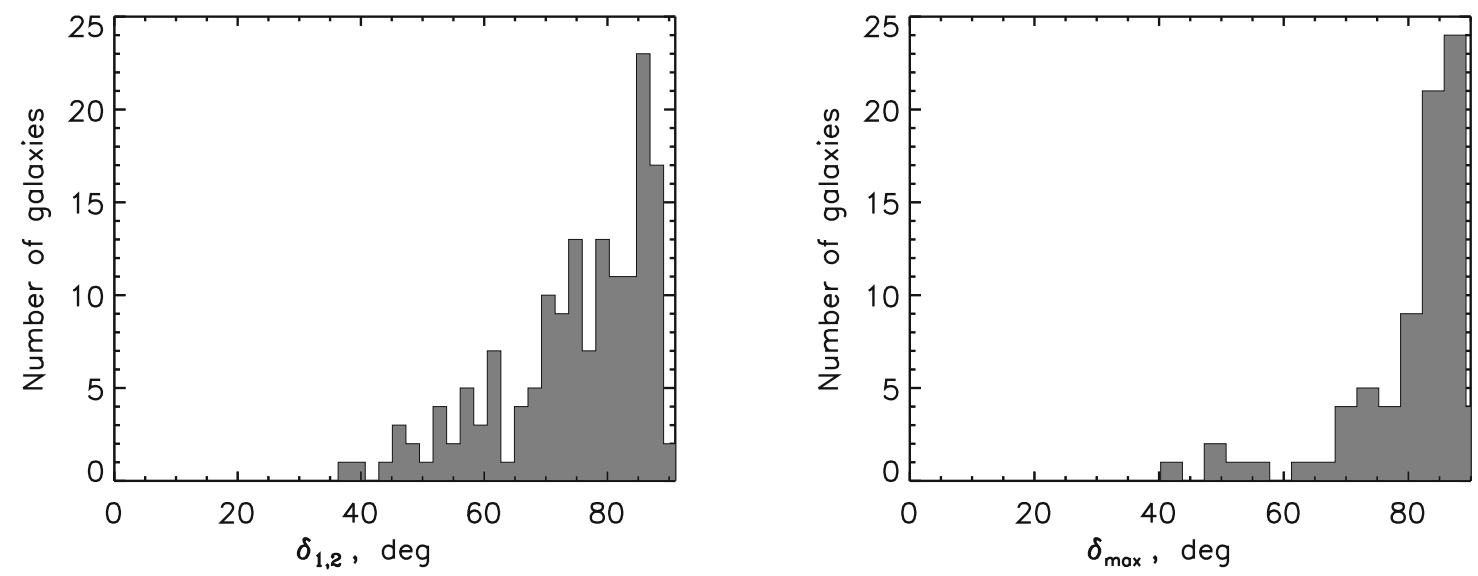

Fig. 2. Histogram of distribution of the angles of inclination of the polar structure to the galactic plane. Left: all possible solutions by the formula (2). Right: only the maximum values of the angles $\delta_{1,2}$ are taken.

\section{COMPUTATION OF MAJOR STRUCTURAL PARAMETERS}

In order to detect the features of low surface brightness of a ring and a galaxy, we have summarized the images in the five SDSS filters $(u, r, i, z, g)$. Since the PRG images have complex shapes, the use of standard automatic algorithms to fit the isophote shapes is problematic. Therefore, we manually fitted the external isophotes of the central disk and the polar ring by the ellipses, using the tools of the SAOImage DS9 software (see examples in Fig. 1). The center of ellipses was fixed on the core of the galaxy. Measured parameters are the major $(a)$ and minor (b) semiaxes of the ellipse and the position angle of the major axis PA. We also tried to describe the inner borders of polar rings but managed to obtain a consistent result for less than a dozen of galaxies, and hence the analysis of these measurements was not done. For the object SPRC 33 (NGC 4262), which is a lenticular galaxy surrounded by a wide gaseous ring hosting only a small number of stars visible mainly in the UV range, the parameters of the ring and the galaxy were taken from [11].

\subsection{Inclination Angles}

To determine the angle of inclination of the studied structures to the line of sight $(i)$, we used the classical formula for estimating the oblate spheroid inclination from the apparent relation of its axes, projected on the celestial sphere:

$$
\cos ^{2} i=\frac{(b / a)^{2}-q_{0}^{2}}{1-q_{0}^{2}},
$$

where the intrinsic flattening of the spheroid for the central galaxies was assumed to be $q_{0}=0.20$ [12], while the polar structures were considered to be flat: $q_{0}=0$.
Knowing only PA and $i$ for the two planes, with the angle $i$ measured by formula (1), we cannot unambiguously determine the angle therebetween. Additional information as of which side of the disk and the ring is closer/farther from the observer is yet required. This can be conceived from the distribution of the dust lanes in the image of the galaxy, but in the vast majority of studied objects their angular size is too small compared to the resolution of SDSS images. In general, there are two solutions for the possible angle between the components of the ring and the galaxy [13]. These values are denoted below as $\delta_{1}$ and $\delta_{2}$ :

$$
\begin{aligned}
\cos \delta_{1,2}= & \pm \cos \left(\mathrm{PA}_{0}-\mathrm{PA}_{1}\right) \sin i_{0} \sin i_{1} \\
& +\cos i_{0} \cos i_{1} .
\end{aligned}
$$

Here $\mathrm{PA}_{0}$ is the position angle of the central galaxy, $\mathrm{PA}_{1}$ is the position angle of the polar ring, $i_{0}$ and $i_{1}$ are the angles of inclination of the galaxy and of the polar ring to the line of sight, respectively. The results are listed in the table.

Figure 2 shows the distributions of the angle between the outer ring and the disk for our sample. The left histogram shows the distribution for all possible values of $\delta$ from (2). Despite the uncertainty in the estimates of $\delta$, the outer ring structures mostly prove to be oriented close to the polar plane: $\delta \geq 70^{\circ}$. This means that the polar configuration in PRGs occurs much more frequently than the inclined one. This is consistent with the theoretical studies, which reveal the existence of stable orbits in the case of axially symmetric (but not spherical) or triaxial distribution of gravitational potential in the polar plane. At the same time, the orbits in the plane notably different from polar prove to be unstable: the stellar ring is 
Galaxy parameters

\begin{tabular}{|c|c|c|c|c|c|c|c|c|c|c|}
\hline \multirow{2}{*}{ Name } & \multicolumn{3}{|c|}{ Inner disk } & \multicolumn{3}{|c|}{ Polar ring } & \multirow{2}{*}{$z$} & \multirow{2}{*}{$\delta_{1}, \operatorname{deg}$} & \multirow{2}{*}{$\delta_{2}$, deg } & \multirow{2}{*}{$D_{\text {ring }} / D_{\text {disk }}$} \\
\hline & $a, \operatorname{arcsec}$ & $b, \operatorname{arcsec}$ & PA, deg & $a, \operatorname{arcsec}$ & $b$, arcsec & PA, deg & & & & \\
\hline SPRC 1 & 7.2 & 3.6 & 92 & 5.6 & 2.4 & 10 & 0.073 & 71 & 84 & 0.78 \\
\hline SPRC 2 & 10.7 & 7.5 & 17 & 19.8 & 5.9 & 85 & 0.035 & 62 & 86 & 1.85 \\
\hline SPRC 3 & 5.7 & 2.8 & 325 & 12.1 & 2.9 & 66 & 0.037 & 86 & 74 & 2.11 \\
\hline SPRC 4 & 9.1 & 3.7 & 122 & 9.5 & 4.0 & 27 & 0.043 & 85 & 77 & 1.04 \\
\hline SPRC 5 & 4.0 & 3.2 & 220 & 8.7 & 2.0 & 106 & 0.028 & 86 & 65 & 2.20 \\
\hline SPRC 6 & 4.8 & 3.2 & 29 & 6.1 & 2.8 & 149 & 0.019 & 87 & 50 & 1.28 \\
\hline SPRC 7 & 4.2 & 3.2 & 150 & 17.8 & 9.5 & 50 & 0.060 & 72 & 60 & 4.28 \\
\hline SPRC 8 & 5.2 & 2.8 & 130 & 7.6 & 2.4 & 27 & - & 88 & 69 & 1.46 \\
\hline SPRC 9 & 5.7 & 1.9 & 10 & 5.5 & 1.4 & 94 & 0.145 & 80 & 88 & 0.97 \\
\hline SPRC 10 & 5.7 & 1.8 & 320 & 6.9 & 1.0 & 40 & 0.042 & 78 & 82 & 1.21 \\
\hline SPRC 11 & 6.7 & 3.2 & 125 & 10.4 & 3.5 & 30 & 0.066 & 85 & 77 & 1.56 \\
\hline SPRC 12 & 4.0 & 2.3 & 135 & 6.3 & 2.0 & 37 & 0.063 & 86 & 73 & 1.60 \\
\hline SPRC 13 & 8.3 & 3.2 & 15 & 13.1 & 2.4 & 97 & 0.032 & 79 & 86 & 1.57 \\
\hline SPRC 14 & 8.7 & 5.2 & 130 & 17.8 & 5.9 & 35 & 0.032 & 83 & 75 & 2.05 \\
\hline SPRC 15 & 9.9 & 4.4 & 144 & 11.9 & 4.0 & 22 & 0.034 & 71 & 53 & 1.20 \\
\hline SPRC 16 & 5.5 & 2.5 & 119 & 10.7 & 2.4 & 48 & 0.060 & 67 & 78 & 1.93 \\
\hline SPRC 17 & 10.0 & 4.4 & 100 & 7.6 & 2.4 & 10 & 0.026 & 82 & 82 & 0.76 \\
\hline SPRC 18 & 4.2 & 2.8 & 45 & 5.5 & 2.6 & 150 & 0.082 & 82 & 61 & 1.33 \\
\hline SPRC 19 & 4.8 & 2.4 & 109 & 5.9 & 1.8 & 12 & 0.105 & 87 & 75 & 1.25 \\
\hline SPRC 20 & 5.1 & 4.0 & 0 & 17.0 & 1.6 & 97 & 0.074 & 89 & 81 & 3.34 \\
\hline SPRC 21 & 3.6 & 2.2 & 9 & 4.8 & 2.0 & 99 & 0.081 & 75 & 75 & 1.33 \\
\hline SPRC 22 & 3.4 & 2.6 & 70 & 7.3 & 2.0 & 335 & 0.160 & 81 & 75 & 2.18 \\
\hline SPRC 23 & 9.9 & 6.7 & 65 & 13.1 & 5.2 & 4 & 0.028 & 53 & 85 & 1.32 \\
\hline SPRC 24 & 8.3 & 7.1 & 80 & 17.8 & 5.7 & 340 & 0.047 & 79 & 68 & 2.14 \\
\hline SPRC 25 & 3.6 & 2.0 & 89 & 7.9 & 1.6 & 4 & 0.073 & 79 & 88 & 2.22 \\
\hline SPRC 26 & 3.8 & 2.4 & 177 & 6.7 & 1.2 & 99 & - & 74 & 86 & 1.79 \\
\hline SPRC 27 & 5.9 & 2.7 & 125 & 14.3 & 2.2 & 30 & 0.048 & 89 & 81 & 2.40 \\
\hline SPRC 28 & 6.0 & 3.2 & 60 & 5.4 & 2.0 & 320 & 0.077 & 87 & 70 & 0.90 \\
\hline SPRC 29 & 5.6 & 4.0 & 0 & 7.9 & 2.0 & 116 & 0.047 & 82 & 61 & 1.43 \\
\hline SPRC 30 & 3.8 & 2.2 & 30 & 3.8 & 1.6 & 130 & 0.075 & 84 & 68 & 1.00 \\
\hline SPRC 31 & 7.2 & 5.2 & 105 & 14.0 & 2.4 & 358 & 0.050 & 85 & 71 & 1.94 \\
\hline SPRC 32 & 5.3 & 3.0 & 5 & 4.8 & 1.3 & 103 & 0.035 & 88 & 75 & 0.89 \\
\hline SPRC 33 & 55.0 & 47.0 & 0 & 120.0 & 50.0 & 25 & 0.005 & 37 & 85 & 2.18 \\
\hline
\end{tabular}


Galaxy parameters. (Contd.)

\begin{tabular}{|c|c|c|c|c|c|c|c|c|c|c|}
\hline \multirow{2}{*}{ Name } & \multicolumn{3}{|c|}{ Inner disk } & \multicolumn{3}{|c|}{ Polar ring } & \multirow{2}{*}{$z$} & \multirow{2}{*}{$\delta_{1}$, deg } & \multirow{2}{*}{$\delta_{2}$, deg } & \multirow{2}{*}{$D_{\text {ring }} / D_{\text {dist }}$} \\
\hline & $a$, arcsec & $b, \operatorname{arcsec}$ & PA, deg & $a, \operatorname{arcsec}$ & $b$, arcsec & PA, deg & & & & \\
\hline SPRC 34 & 5.3 & 3.0 & 343 & 9.9 & 1.6 & 73 & 0.081 & 85 & 85 & 1.85 \\
\hline SPRC 35 & 5.3 & 1.7 & 115 & 9.1 & 1.3 & 15 & 0.068 & 82 & 78 & 1.70 \\
\hline SPRC 36 & 5.9 & 2.4 & 15 & 4.4 & 1.6 & 115 & - & 88 & 73 & 0.73 \\
\hline SPRC 37 & 4.4 & 2.4 & 134 & 6.3 & 2.0 & 39 & 0.068 & 84 & 76 & 1.45 \\
\hline SPRC 38 & 11.1 & 4.8 & 115 & 15.8 & 5.9 & 12 & 0.039 & 87 & 70 & 1.43 \\
\hline SPRC 39 & 7.1 & 4.0 & 115 & 15.8 & 3.6 & 50 & 0.029 & 62 & 76 & 2.22 \\
\hline SPRC 40 & 47.5 & 13.1 & 105 & 26.9 & 5.9 & 57 & 0.004 & 46 & 53 & 0.57 \\
\hline SPRC 41 & 9.3 & 3.8 & 342 & 9.1 & 2.6 & 100 & 0.061 & 71 & 58 & 0.98 \\
\hline SPRC 42 & 8.5 & 6.1 & 10 & 51.5 & 5.6 & 60 & 0.023 & 58 & 67 & 6.04 \\
\hline SPRC 43 & 4.8 & 3.2 & 10 & 7.5 & 1.8 & 67 & 0.171 & 56 & 75 & 1.58 \\
\hline SPRC 44 & 5.9 & 2.4 & 49 & 5.2 & 2.6 & 320 & 0.113 & 79 & 80 & 0.87 \\
\hline SPRC 45 & 4.8 & 4.0 & 0 & 8.8 & 2.4 & 85 & 0.072 & 74 & 79 & 1.83 \\
\hline SPRC 46 & 4.9 & 2.0 & 72 & 4.8 & 1.6 & 7 & 0.128 & 60 & 75 & 0.96 \\
\hline SPRC 47 & 31.7 & 5.6 & 110 & 27.7 & 7.1 & 360 & 0.031 & 70 & 70 & 0.88 \\
\hline SPRC 48 & 8.7 & 5.2 & 23 & 13.9 & 3.6 & 123 & 0.056 & 89 & 73 & 1.59 \\
\hline SPRC 49 & 9.9 & 5.9 & 310 & 15.8 & 6.9 & 43 & 0.068 & 77 & 73 & 1.60 \\
\hline SPRC 50 & 8.7 & 5.3 & 40 & 31.7 & 4.0 & 2 & 0.078 & 45 & 56 & 3.64 \\
\hline SPRC 51 & 4.2 & 2.0 & 7 & 6.7 & 2.8 & 90 & 0.075 & 73 & 85 & 1.62 \\
\hline SPRC 52 & 21.6 & 4.6 & 80 & 15.8 & 4.0 & 146 & 0.015 & 65 & 67 & 0.73 \\
\hline SPRC 53 & 5.1 & 2.2 & 312 & 4.0 & 1.8 & 48 & 0.083 & 85 & 74 & 0.78 \\
\hline SPRC 54 & 5.3 & 4.3 & 45 & 14.6 & 2.2 & 334 & 0.039 & 71 & 85 & 2.74 \\
\hline SPRC 55 & 4.8 & 3.6 & 0 & 13.9 & 1.5 & 85 & 0.086 & 82 & 88 & 2.92 \\
\hline SPRC 56 & 13.2 & 11.6 & 120 & 16.0 & 5.6 & 338 & 0.055 & 86 & 48 & 1.21 \\
\hline SPRC 57 & 3.6 & 2.6 & 145 & 5.2 & 1.8 & 27 & 0.070 & 86 & 56 & 1.45 \\
\hline SPRC 58 & 7.6 & 7.2 & 103 & 24.0 & 3.2 & 332 & - & 85 & 70 & 3.16 \\
\hline SPRC 59 & 4.5 & 3.5 & 70 & 12.3 & 2.6 & 343 & - & 78 & 82 & 2.73 \\
\hline SPRC 60 & 3.4 & 3.2 & 135 & 9.9 & 2.4 & 55 & 0.078 & 73 & 80 & 2.94 \\
\hline SPRC 61 & 8.4 & 5.2 & 49 & 12.0 & 2.4 & 320 & 0.046 & 82 & 83 & 1.43 \\
\hline SPRC 62 & 2.6 & 2.4 & 45 & 5.2 & 1.7 & 45 & - & 48 & 86 & 2.00 \\
\hline SPRC 63 & 2.4 & 1.8 & 130 & 4.3 & 1.4 & 60 & 0.074 & 62 & 88 & 1.79 \\
\hline SPRC 64 & 3.1 & 2.2 & 350 & 5.2 & 1.6 & 101 & - & 87 & 62 & 1.67 \\
\hline SPRC 65 & 11.9 & 2.6 & 92 & 9.9 & 3.2 & 310 & 0.067 & 44 & 39 & 0.83 \\
\hline SPRC 66 & 8.8 & 4.4 & 33 & 9.2 & 2.8 & 117 & 0.087 & 76 & 86 & 1.05 \\
\hline
\end{tabular}


Galaxy parameters. (Contd.)

\begin{tabular}{|c|c|c|c|c|c|c|c|c|c|c|}
\hline \multirow{2}{*}{ Name } & \multicolumn{3}{|c|}{ Inner disk } & \multicolumn{3}{|c|}{ Polar ring } & \multirow{2}{*}{$z$} & \multirow{2}{*}{$\delta_{1}, \operatorname{deg}$} & \multirow{2}{*}{$\delta_{2}$, deg } & \multirow{2}{*}{$D_{\text {ring }} / D_{\text {disk }}$} \\
\hline & $a$, arcsec & $b$, arcsec & PA, deg & $a$, arcsec & $b$, arcsec & PA, deg & & & & \\
\hline SPRC 67 & 18.6 & 4.4 & 113 & 25.7 & 3.2 & 347 & 0.028 & 55 & 53 & 1.38 \\
\hline SPRC 68 & 15.8 & 4.8 & 30 & 7.9 & 4.8 & 115 & - & 78 & 86 & 0.50 \\
\hline SPRC 69 & 5.9 & 3.6 & 135 & 14.7 & 2.8 & 32 & 0.025 & 85 & 73 & 2.47 \\
\hline SPRC 70 & 5.2 & 2.4 & 100 & 9.1 & 3.0 & 13 & 0.069 & 79 & 84 & 1.77 \\
\hline SPRC 241 & 13.9 & 6.7 & 9 & 27.7 & 15.8 & 110 & 0.018 & 83 & 66 & 2.00 \\
\hline SPRC 260 & 16.6 & 7.9 & 82 & 27.7 & 15.8 & 15 & 0.021 & 57 & 87 & 1.67 \\
\hline PRC A3 & 60.0 & 32.0 & 38 & 46.0 & 18.0 & 110 & 0.003 & 63 & 87 & 0.77 \\
\hline PRC A4 & 14.3 & 7.9 & 133 & 43.6 & 4.4 & 53 & 0.023 & 78 & 84 & 3.05 \\
\hline PRC A6 & 15.8 & 7.9 & 125 & 49.5 & 7.9 & 17 & 0.018 & 78 & 69 & 3.13 \\
\hline PRC B9 & 15.8 & 10.7 & 5 & 21.4 & 5.9 & 69 & 0.020 & 60 & 82 & 1.35 \\
\hline PRC B 17 & 26.0 & 10.0 & 135 & 32.0 & 6.0 & 24 & 0.004 & 74 & 66 & 1.23 \\
\hline PRC C13 & 122.8 & 39.2 & 40 & 277.2 & 59.4 & 352 & 0.003 & 46 & 54 & 2.26 \\
\hline
\end{tabular}

destroyed by the differential precession [14], and the gas ring rather quickly (in several revolutions) settles into one of the principal planes of the central galaxy (see, e.g., [15]). Of course, the lifetime and the nature of the evolution of the inclined ring depends on the shape of distribution of the potential $([16,17]$ and references therein).

If we, in agreement with the above theoretical calculations, assume that out of the two possible values $\delta_{1}$ and $\delta_{2}$ the most likely is the angle closer to $90^{\circ}$, then the distribution of inclination angles of rings to the disk will take the shape shown in Fig. 2 to the right. The peak of the distribution becomes considerably narrower, in such a way that for $95 \%$ of all sample objects $\delta>70^{\circ}$. Two important points should be noted.

- Even with this "optimistic" assumption about the implementation of cases of large angles only, $6 \%$ of the sample (five objects) are still galaxies with moderately inclined external structures: $\delta=40-55^{\circ}$. It is possible that they are not stable and we have caught them in the process of destruction (sedimentation in a plane disk). Detailed modeling of the characteristics of these specific galaxies may yield the answer.

- A precisely polar orientation (within $5^{\circ}$ from the plane orthogonal to the disk) is observed only in 39 galaxies.
It should of course be borne in mind that the calculation of angles of inclination via formula (1) implies that the polar structures are flat and round. At the same time, there are several indications that, firstly, polar rings must possess weak internal ellipticity [18] and, secondly, the structures deviating from the polar plane have to warp [14], and hence it is not always correct to attribute them a single angle. However, we believe that the above-mentioned statistical regularities should be explained within the theoretical calculations.

In general, the given distributions by the angle $\delta$ are similar to Fig. 3 from the earlier paper by Whitmore [9], where the author also makes a conclusion that the observed distribution of the inclination angle of the ring to the disk is explained by the preference of the polar spatial orientation. At the same time, analyzing a few times larger sample of PRGs, we cannot agree with the conclusion of [9] that the absence of rings with a moderate inclination (in our terms it means $\delta<55^{\circ}$ ) is only due to the selection effect. On the one hand, indeed, during the visual search of PRG candidates, the objects in which both components are visible at a large angle to the line of sight prove to be more noticeable: the average value of the angle $i_{0}$ in our sample is $56^{\circ}$. Moreover, polar structures are visible at a yet larger angle: the average $i_{1}$ is $70^{\circ}$, given that $i_{1}<60^{\circ}$ only for eight rings. However, even if we only select the objects visible edge-on, then from (2) for $i_{0} \approx i_{1} \approx 90^{\circ}$ we get $\cos \delta \approx \cos \left(\mathrm{PA}_{0}-\mathrm{PA}_{1}\right)$. Hence, moderately inclined 

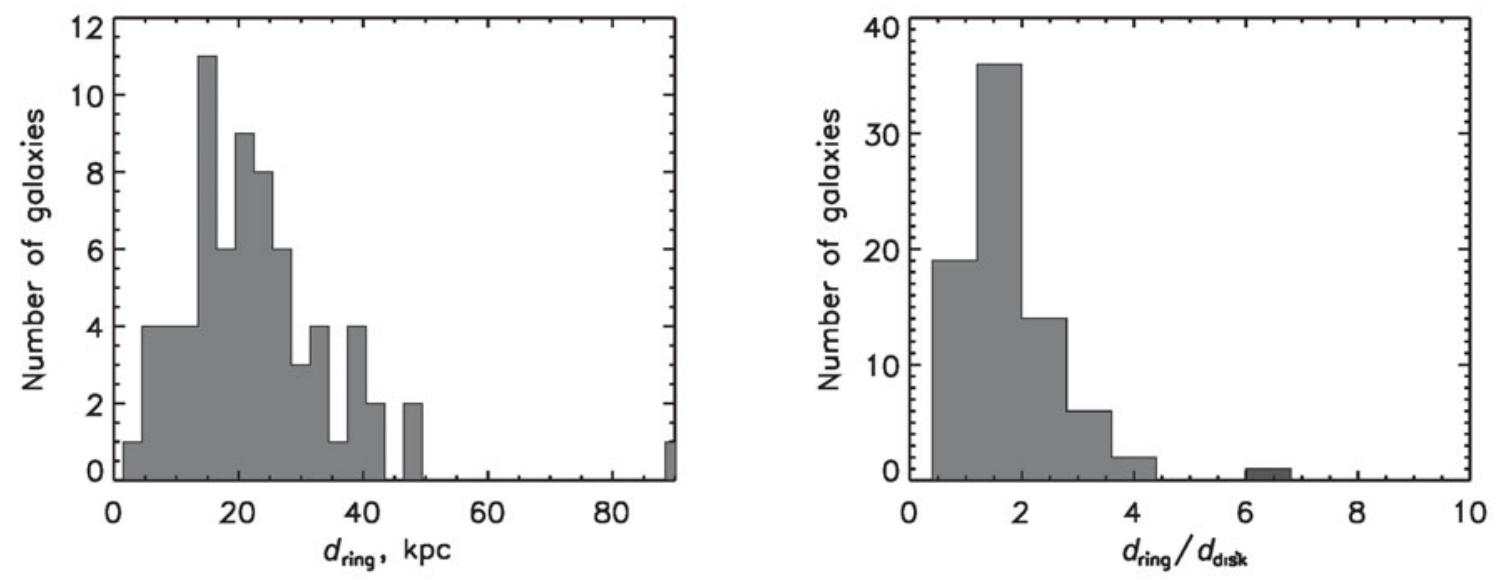

Fig. 3. Distributions of the outer diameters of polar structures. Left: linear size in kpc. A giant ring SPRC 50 with the diameter of $90 \mathrm{kpc}$ stands out from the total distribution. Right: the diameter of the rings is normalized to the size of the disk of the central galaxy.

rings would attract attention by the corresponding difference in the position angles. Therefore, the selection effect cannot make the observed distributions of $\delta$ flatter than those shown in Fig. 2.

\subsection{Spatial Scales}

The calculations of linear diameters were performed for $H_{0}=71 \mathrm{~km} / \mathrm{s} / \mathrm{Mpc}$ from the known redshifts of galaxies, listed in paper [1], where they, in turn, were taken from the SDSS and NED. Figure 3 shows a histogram of distribution of the outer diameters of rings. Although the scatter in their values is quite large, almost all the rings are smaller than $40 \mathrm{kpc}$ at an average diameter of about $20 \mathrm{kpc}$. Importantly, since the measurements were done from the optical images, this is the case of stellar polar structures only. In neutral gas the size of the outer structures can be much larger, and the inclination to the plane of the disk may vary. For example, the distribution of $\mathrm{HI}$ in the PRC A3 (NGC 2685) galaxy agrees with the stellar polar ring in the inner regions, but at large radii it warps the way that it again fits in the plane of the main galaxy [19].

SPRC 50 largely excels from the total distribution, its image is shown in Fig. 1. The central galaxy is surrounded by a giant disk of low surface brightness. The spectrum of the core of SDSS DR7 reveals absorption features characteristic of early-type galaxies at redshift $z=0.078$ which corresponds to the scale of $1.5 \mathrm{kpc} /$ arcsec. If the polar disk is not a result of a random projection, its diameter reaches $90 \mathrm{kpc}$. This is comparable with the sizes of the most extended galactic disks known to date, such as Malin 1 (90 kpc in the diameter, according to [20]), the prototype of the low surface brightness galaxies, or a ring galaxy UGC 7069 (115 kpc, according to [21]). The existence of such giant polar structures itself is a serious challenge to the theories of galaxy formation, unless, of course, spectral observations confirm that SPRC 50 is not a random projection but a real $P R G$.

Figure 3 (right) shows the distribution of the outer diameters of the rings, normalized to the optical diameter of the galaxy: $d_{\text {ring }} / d_{\text {disk }}$. Note that the outer boundaries of the low brightness regions measured by our method correspond to the traditional criterion of the size of the disk by the level of $25^{\mathrm{m}}$ from a square arcsec $\left(D_{25}\right)$. For the majority of galaxies, the diameter of the ring does not exceed from three to four diameters of the disk, with the average of about $d_{\text {ring }} / d_{\text {disk }} \approx 1$.7. In this distribution there is a galaxy with an unusually large relative size of the ring, SPRC 42. Its image is shown in Fig. 1 to the right. The diameter of the polar component, visible edgeon with slightly warped outer parts makes up about $47 \mathrm{kpc}$. Such a long structure surrounding a relatively small lenticular galaxy should be more correctly called the "polar disk." We are now conducting additional observations of this intriguing object in the optical and radio bands.

In $22 \%$ of the sample ( 17 galaxies) $d_{\text {ring }} / d_{\text {disk }} \leq 1$. The entire bright part of these objects' polar rings rotates crossing the disk of the host galaxy. It is clear that the spatial density of stars is small and direct collisions of stellar components do not occur. But if the polar ring contains gas, shock waves may be generated while the gas clouds pass through the gravitational well of the stellar disk. Moreover, if the inner disk contains its own gas, direct collision of gas flows becomes possible. Unfortunately, literature has 

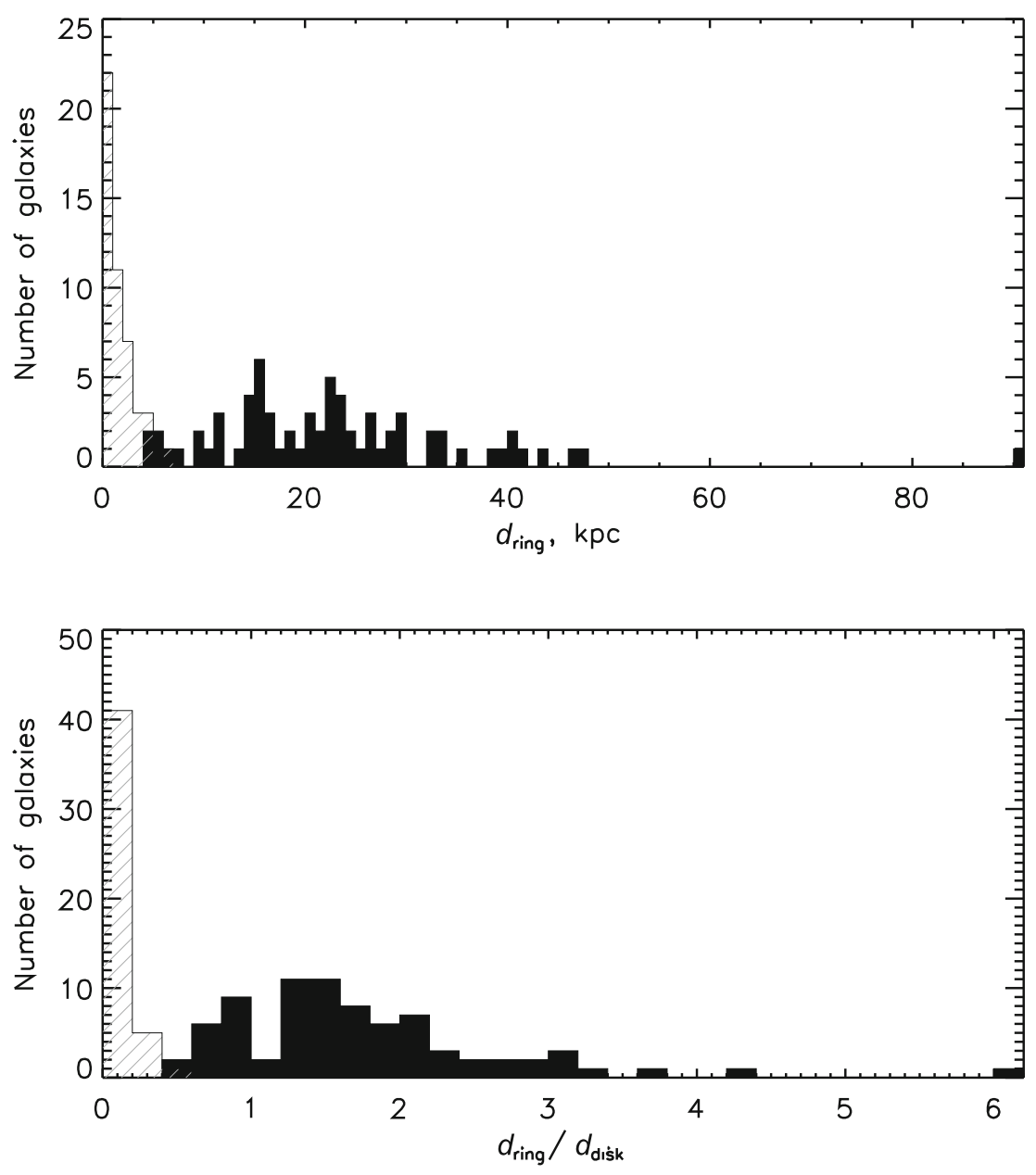

Fig. 4. The distributions of polar structures by the diameter. Top-linear sizes, bottom-normalization to the diameter of the central disk. Black color in the figure denotes the outer structures, hatching - the inner rings from [22].

almost no theoretical considerations of the process, with the exception of one article by Wakamatsu [23]. It is possible that exactly such a picture, i.e., impact ionization of gas on inclined orbits is observed in NGC 7743. This is a lenticular galaxy, in which the entire ionized gas at $r=1.5-5.4 \mathrm{kpc}$ is located in the plane inclined by $34^{\circ}$ or $77^{\circ}$ to the stellar disk [24].

It is interesting to compare the distributions in Fig. 3 with those for the inner polar rings and disks observed in the circumnuclear regions of a number of nearby galaxies. Figure 4, in addition to the already considered distributions of the diameters and relative diameters of the outer polar structures, shows the data on 47 galaxies with confirmed inner structures from [22]. For the inner polar structures, the diameter of the disk of the galaxy was accepted as the diameter of $D_{25}$ taken from the NED database. We can see that the sizes of inner and outer polar structures form a continuous sequence from a hundred parsecs to tens of kiloparsecs. By the values of normalized diameters both samples come into contact at $d_{\text {ring }} / d_{\text {disk }} \approx 0.5$, hence the galaxy NGC 5014 (SPRC 40) is included in both lists: inner structures and SPRC catalog. At the same time, a relative shortage of "intermediate size" polar structures $\left(d_{\text {ring }} / d_{\text {disk }} \approx 0.4-0.7\right)$ is noticeable. Only two galaxies from the SDSS sample find themselves here: SPRC 40 and SPRC 68.

Such a bimodal distribution of the relative size of polar structures is most likely due to the fact that in all the cases considered, the stability of the polar orbit is controlled by the spheroidal or triaxial gravitational potential. But if the gravitational potential of the dark halo is important for the stability of large-scale structures, the bulge (including the triaxial one) or the central bar play the dominant role for the inner rings and disks. In the intermediate zone of radial scales, the shape of the potential is markedly different from the spheroidal/triaxial one, since a significant contri- 


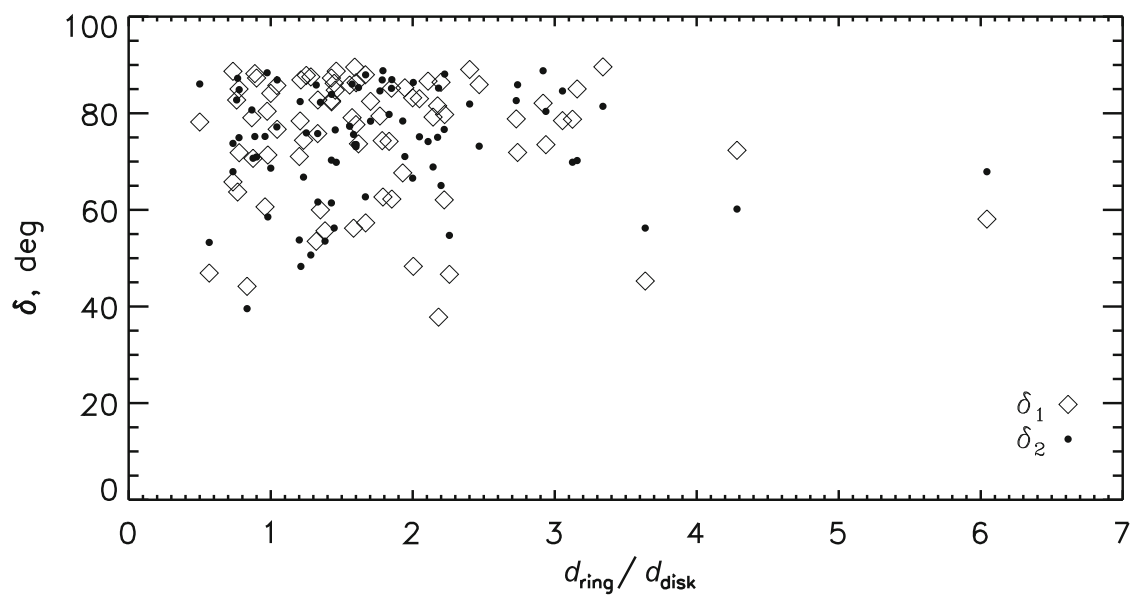

Fig. 5. Dependence of the inclination angles on the diameter of outer polar structures, normalized to the size of the galaxy. The angles $\delta_{1}, \delta_{2}$ are marked by different symbols.

bution in the gravitational potential is brought here by the flat stellar disk. Therefore, the polar/inclined orbits cease to be stable. Collisions occurring while the disk is crossed by the matter on polar orbits, mentioned in the previous chapter, is the manifestation of the same effect. Apparently it is not a coincidence that in the SPRC 40 the "polar" structure of intermediate size is strongly inclined to the galactic disk and is possibly not stationary.

Figure 5 shows the distribution of points in the plane $\left(d_{\text {ring }} / d_{\text {disk }}, \delta\right)$. Although the scatter of the observed values is quite large, it is indeed clear that the deviation from the polar plane of $\left(\delta=90^{\circ}\right)$ is more frequently observed for the rings crossing the central disk: $d_{\text {ring }} / d_{\text {disk }}<1$. The largest rings are as well revealing the drifts from the polar plane $\left(d_{\text {ring }} / d_{\text {disk }}>3.5\right)$ which is apparently related to the development of warp instability at decreasing halo density.

\section{CONCLUSIONS}

We have considered a sample of 78 most reliable polar ring galaxy candidates, represented in the SDSS survey, some of which already have kinematic confirmations. Most of the outer structures are indeed polar, i.e., inclined at an angle of more than $70^{\circ}$ to the central plane of the galaxy. We show that the distribution of relative sizes of the outer polar rings and circumnuclear polar disks (inner rings) is bimodal, with a "drop" falling at the interval of $0.4<d_{\text {ring }} / d_{\text {disk }}<0.7$. This is most likely due to the fact that polar and inclined structures of intermediate sizes prove to be short-lived. At that, the stability of inner polar rings and disks is supported by the gravity of the bulge, while the outer structures are maintained by the gravity of the dark halo.

\section{ACKNOWLEDGMENTS}

This work was supported by the grants of the Russian Foundation for Basic Research (project no. 13-02-00416-a) and the "Active Processes in Galactic and Extragalactic Objects" basic research program of the Department Physical Sciences of the RAS OFN-17. A. Moiseev thanks the non-profit Dynasty foundation for the support. In the process of work we used the NASA/IPAC (NED) database of extragalactic data, managed by the Jet Propulsion Laboratory of the California Institute of Technology under the contract with the NASA (USA). We also used the public data of the SDSS project supported by the Alfred P. Sloan foundation, participating institutions of the SDSS collaboration, the National Science Foundation, and the Ministry of Energy of the USA.

\section{REFERENCES}

1. A. V. Moiseev, K. I. Smirnova, A. A. Smirnova, and V. P. Reshetnikov, Monthly Notices Roy. Astronom. Soc. 418, 244, (2011).

2. B. C. Whitmore, R. A. Lucas, D. B. McElroyet, et al., Astronom. J. 100, 1489 (1990).

3. F. Bournaud and F. Combes, Astronom. and Astrophys. 401, 817 (2003).

4. F. Combes, EAS Pub. Ser. 20, 97 (2006).

5. V. Reshetnikov and N. Sotnikova, Astronom. and Astrophys. 325, 933 (1997).

6. A. V. Maccio, B. Moore, and J. Stabel, Astronom. J. 636, 25 (2006).

7. C. B. Brook and F. Governato, Astronom. J. 689, 678 (2008).

8. M. Spavone, E. Iodice, M. Arnaboldi, et al., Astronom. J. 714, 1081 (2010). 
9. B. C. Whitmore, in Warped Disks and Inclined Rings Around Galaxies, Ed. by S. Casertano, P. D. Sackett, and F. H. Briggs (Cambridge University Press, 1991), p. 60.

10. S. Khoperskov, A. Moiseev, and A. Khoperskov, Memorie della Societa Astronomica Italiana Suppl. 25, 51 (2013).

11. S. A. Khoperskov et al., submitted to Monthly Notices Roy. Astronom. Soc.

12. E. Hubble, Astrophys. J. 64, 321 (1926).

13. A. V. Moiseev, Astrophysical Bulletin 63, 201 (2008).

14. L. S. Sparke, Monthly Notices Roy. Astronom. Soc. 219,657 (1986).

15. A. Habe and S. Ikeuchi, Astrophys. J. 289, 540 (1985).

16. R. F. Peletier and D. M. Christodoulou, Astronom. J. 105, 1378 (1993).
17. M. Ideta, S. Hozumi, T. Tsuchiya, and M. Takizawa, Monthly Notices Roy. Astronom. Soc. 311, 733 (2000).

18. E. Iodice, F. Bournaud, F. Combes, et al., Astrophys. J. 585, 730 (2003).

19. G. I. G. Józsa, Astronom. and Astrophys. 494, 489 (2009).

20. G. D. Bothun, Ch. D. Impey, D. Malin, and J. R. Mould, Astronom. J. 94, 23 (1987).

21. K. K. Ghosh and M. Mapelli, Monthly Notices Roy. Astronom. Soc. 386, L38 (2008).

22. A. V. Moiseev, Astrophysical Bulletin 67, 147 (2012).

23. K. Wakamatsu, Astronom. J. 105, 1745 (1993).

24. I. Yu. Katkov, A. V. Moiseev, and O. K Sil'chenko, Astrophys. J. 740, 83 (2011). 